\title{
Unsupervised Identification and Prediction of Foothold Robustness
}

\author{
M. A. Hoepflinger, M. Hutter, C. Gehring, M. Bloesch, and R. Siegwart, Fellow, IEEE
}

\begin{abstract}
This paper addresses the problem of evaluating and estimating the mechanical robustness of footholds for legged robots in unstructured terrain. In contrast to approaches that rely on human expert knowledge or human defined criteria to identify appropriate footholds, our method uses the robot itself to assess whether a certain foothold is adequate or not. To this end, one of the robot's legs is employed to haptically explore an unknown foothold. The robustness of the foothold is defined by a simple metric as a function of the achievable ground reaction forces. This haptic feedback is associated with the foothold shape to estimate the robustness of untouched footholds. The underlying shape clustering principles are tested on synthetic data and in hardware experiments using a singleleg testbed.
\end{abstract}

\section{INTRODUCTION}

Over the past years, legged robotics has made impressive progress with respect to locomotion on irregular and highly unstructured terrain. Walking systems have started to be able to navigate in surroundings in which they can actually show their inherent advantages in comparison to wheeled vehicles. Such locomotion in rough terrain is extremely challenging and poses a great number of problems in the areas of planning, perception, and control. For example, it requires sophisticated balance controllers, adaptive gait patterns, as well as reliable foothold selection methods. The latter is particularly critical, since it determines the robustness and achievable speed of legged locomotion in rough terrain. Groundbreaking research results in this domain were recently achieved within the DARPA Learning Locomotion Challenge. In this project, the participants had to traverse a terrain sample with a small quadrupedal robot (LittleDog from Boston Dynamics, [1]) as fast as possible. The outcome of the competition therefore strongly depended on the appropriate selection of footholds. The small number of terrain samples and a priori knowledge about the possible shape allowed to employ human expert trained or off-line tuned foothold selection methods. One team [2] used hard-coded, human defined criteria such as distances between the foothold center and cliffs or holes, as well as geometric properties that have been hand-tuned to optimize the performance. Other methods characterized the quality of footholds based on decisions of human experts. In [3], for example, an approach is presented in which a set of foothold templates and a corresponding ranking function has been learned based on expert demonstrated

This research was supported by the Swiss National Science Foundation through the National Centre of Competence in Research Robotics, (NCCR).

M. A. Hoepflinger, M. Hutter, C. Gehring, M. Bloesch, and R. Siegwart are with the Autonomous Systems Lab, Institute for Robotics and Intelligent Systems, Swiss Federal Institute of Technology (ETHZ), 8092 Zurich, Switzerland (+41 4463221 45; fax: +41 4463211 81; e-mail: markho@ethz.ch footholds, and in [4], a number of pairs of terrain samples have been evaluated against each other by a human expert. Based on the preferences of the expert, the system learned a cost function and applied the knowledge for footstep planning. However, apart from the tedious process to train such systems by a human expert, it is often not obvious if the decision made by this expert is the optimal choice from the perspective of the robot. With increasing complexity of the robot and a growing diversity of the terrain samples, it is getting nearly impossible for humans to predict all possible cases such that the robot can select an optimal foothold solely based on pre-programmed knowledge. For this reason, legged robots should possess the ability to assess the quality of a given foothold by themselves without using additional human input. This will eventually allow the robots to continuously learn from their own experience and to adapt to unknown and unpredicted environmental challenges, which was extensively studied for wheeled service robots and rough-terrain vehicles ([5] - [8]). As a step towards transferring these capabilities to legged systems, this paper presents a method for automatic foothold characterization, and illustrates the application of this strategy on a robotic leg. The haptically identified foothold robustness is associated with the geometrical properties, which is fundamental to predict the robustness of distant footholds. This project builds upon our previous work on classification of different surface types [9] and ground geometries [10], and will eventually lead to an automated vision guided process that can utilize on-line machine learning to perpetually identify appropriate footholds.

\section{METHOD}

When unsure about the mechanical robustness of a foothold, humans tend to use their swing leg to explore the uncertain spot. After this haptic exploration, we are able to estimate the robustness of the foothold and decide whether to step on this location or not. The acquired knowledge combined with the visual interpretation additionally helps us for future decisions. This methodology can be adapted for robotic devices. This requires, on one hand, the establishment of the physical interaction with the terrain and the introduction of appropriate metrics for the quality of the footholds. On the other hand, once the quality of the foothold is evaluated, the haptic information has to be related to other characteristics of the foothold, such as appearance. This allows to predict the foothold quality without exploring every single footstep and is especially useful for planning future foothold locations. The process can be referred to as near-to-far learning: High-resolution data from short-range 


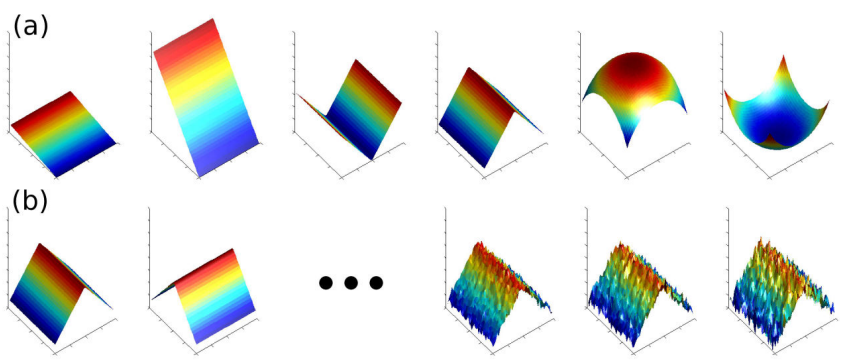

Fig. 1. The upper row contains plots of the six different footholds that are contained in the synthetic foothold data set: flat ground, inclined plane (50 deg), wedge, inverted wedge, convex and concave spherical surface. The lower row illustrates the variations of each foothold (e.g. the wedge). The footholds are rotated and white noise is added.

(e.g. [11]) or data of a different sensor modality (e.g. [5]) are mapped to low-resolution data from a sensor with extended range.

To combine the haptic information with other properties of the footholds, appropriate features that allow to clearly characterize the footholds are required. The first part of the paper deals with the selection of features that permit to distinguish the footholds according to their geometrical properties. The features have to represent the foothold under following invariance criteria: Since we employ a (symmetric) spherical foot, the features have to be rotation invariant as well as invariant to certain level of noise that could be induced by the process of acquiring the foothold properties. To simplify the process of evaluating the effectiveness of those features, a simple synthetic data set of footholds is applied. The data set consists of six different foothold shapes with a total of eight variations such as rotations (to evaluate the invariance to rotations) and corrupted by white noise (Figure 1).

Based on the calculated features, a clustering algorithm is used to group the footholds into distinct categories with similar characteristics. The application of unsupervised learning methods allows to deal with categories that are not a priori defined. To examine the quality of the clustering, internal cluster validation techniques (e.g. [12]) are applied. On the one hand, this helps to evaluate how well the features and clustering methods distinguish the different footholds. On the other hand, it allows to identify the number of clusters that are required to well separate the data.

As a last step, the mechanical robustness of the foothold that is identified by haptic exploration is assigned to the individual clusters respectively the groups of footholds. This step links the geometric properties to the robustness of the foothold and hence allows to predict the quality of unexplored terrain samples purely based on geometric information.

\section{A. Features}

An individual foothold shape is represented as a regularly spaced digital elevation model (DEM) with a resolution of $1 \mathrm{~mm}$ and an edge length, that corresponds to the robotic foot diameter of $4 \mathrm{~cm}$. To consider different spacial scales, the elevation maps of potential footholds are divided into rectangular sub-regions with different edge length (for the experiments: 1, 2, 3 and $4 \mathrm{~cm}$ ). For every sub-region, local features are computed based on the following approaches:

- Feature set $\mathcal{F}_{1}$ : The first set of features is calculated based on features similar to [13] and consist of the standard deviation of the heights, the average slope in $\mathrm{x}$ and $\mathrm{y}$ direction, and finally the minimum/maximum height relative to the center of the foothold. This leads to a total of 20 features per foothold.

- Feature set $\mathcal{F}_{2}$ : The second set of features is based on the computation of the central moments of the elevation map. The central moments are defined as $\mu_{p q}=\sum_{x} \sum_{y}\left(x-x_{c}\right)^{p}\left(y-y_{c}\right)^{q} \cdot h(x, y)$, with $h(x, y)$ the height at coordinates $\mathrm{x}, \mathrm{y}$, and $x_{c}, y_{c}$ the components of the center of gravity. The sum of the heights, the distance between the center of gravity and the center of the foothold (Euclidean norm), the minimal/maximal eigenvalue of the inertial tensor, as well as an indicator for the eccentricity $\epsilon=\frac{\left(\mu_{20}-\mu_{02}\right)^{2}-4 \mu_{11}^{2}}{\left(\mu_{20}+\mu_{02}\right)^{2}}$ are used as features. This results in a total of 20 features, that are computed per foothold.

- Feature set $\mathcal{F}_{3}$ : A further set of features is computed based on Tamura features [14]. The Tamura features are related to human visual perception and reflect the coarseness, contrast, directionality, line-likeness, regularity, and roughness.

\section{B. Clustering algorithm}

Clustering techniques separate data sets into groups with certain similarity. Clustering is an unsupervised method and does therefore not require any prior class identification. To this end, we employ three different algorithms: two hard partitioning methods, namely $K$-means and $K$-medoid, and one fuzzy partitioning method, namely Fuzzy $C$-means. The hard partitioning methods separate a data set into subsets that are disjoint, not empty, and do not contain the complete data set. Each data point is associated to a cluster in order to minimize the "within-cluster distance" with respect to a cluster center. The difference between the K-medoid clustering method and the K-means is the selection of the center that is defined by the nearest data point to the mean of the cluster data in case of the $K$-medoid algorithm. In fuzzy clustering such as Fuzzy $C$-means, a so-called $C$ means functional is minimized [15]. This objective function describes the total variance of the difference between the data object and a potential cluster center.

\section{Internal cluster validation}

Since the structure of the data is not known in advance, internal cluster validation methods (e.g. [16]) are applied to examine how well the data set is separable with respect to its geometrical properties. These are quantitative validation methods that, compared to external validation methods, do not require external information, such as class labels. The evaluation helps to decide on the optimal cluster number 
as well as on other parameters such as on the distance norms or the feature sets. Jain et al. [17] describe the overall process of clustering as a feedback loop, where clustering validation methods are applied to influence the clustering (e.g. by determining an optimal cluster number). To this end, the results of the clustering step are fed back to improve the feature selection as well as the method for clustering validation.

Since objects within the same cluster are required to be similar and objects in different clusters distinct, the validation measures can be outlined by the compactness and the separation of the clusters. Compactness is a measure to express how close the objects of the same group are. The variance as an example, is a common measure for compactness. The separation metric expressed how widely separated the clusters are. Typically, the distances between the cluster centers, the distances between the most distant or closest objects are considered in the computation of a separation metric.

To evaluate the performance of the hard clustering methods, such as $K$-means and $K$-medoid, the following validation metrics were applied:

- the Calinski-Harabasz index $(\mathrm{CH})$ [18] that characterizes the separation and compactness based on the average of the sum of squares within-cluster and betweencluster distances,

- the Dunn's index (DI) [15] that uses the minimum pairwise distance between objects in different clusters for separation evaluation and the maximum distance among all clusters for compactness,

- the Silhouette index (S) [19] that validates the clustering based on the pairwise difference of within-cluster distances and between-cluster distances, and

- the Xie-Beni index (XB) [20] that defines the intercluster separation as the minimum square distance between cluster centers and the compactness as the mean square distance between the data points and their cluster center.

To evaluate the performance of the fuzzy clustering methods, we additionally applied:

- the Partition Coefficient (PC) [21] that reflects the amount of overlap between clusters and

- the Classification Entropy (CE) that indicates the fuzziness of the clusters.

\section{Haptic exploration}

To determine the robustness of a foothold, a robotic foot is brought into contact with the terrain, and the magnitude and direction of the contact force is altered up to a certain predefined level or until slippage is detected by means of kinematic measurements. Interpreting the contact forces as well as the foot position then allows to assign a robustness measure to the foothold. Ideally, the applied contact forces during haptic exploration should be comparable with the forces that are actually generated during walking. Figure 2 illustrates the exploration process. During the first phase of a
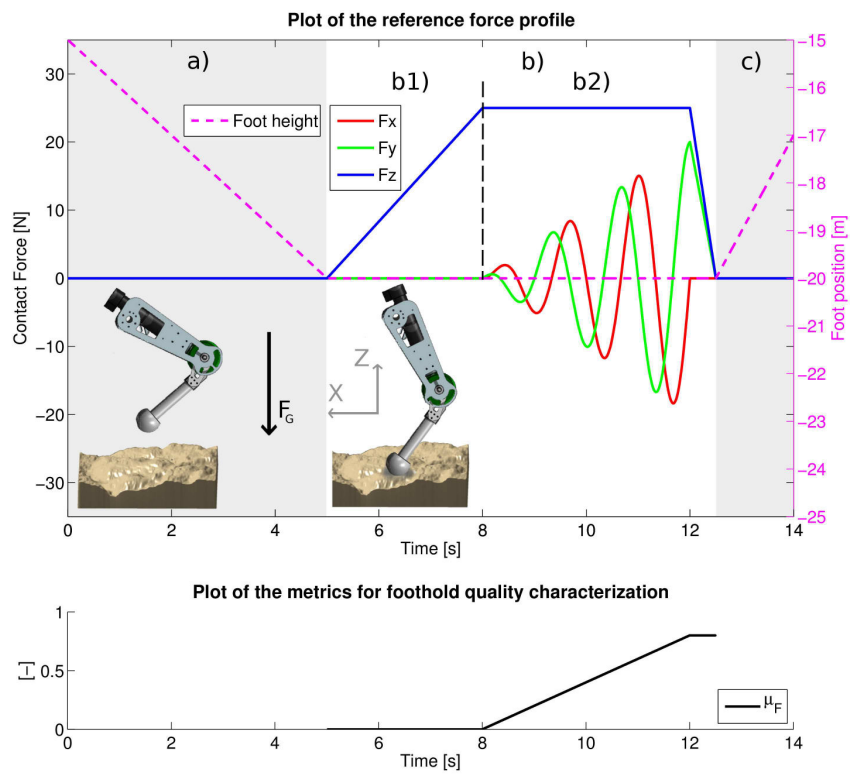

Fig. 2. Upper plot: Example plot of the three phases of the foothold evaluation principle: a) making contact, b) haptic exploration phase, and c) retraction phase. As soon as ground contact is detected, the leg starts the haptic exploration process. Lower plot: The introduced metric $\mu_{F}$ to characterize the quality of the sample foothold.

measurement cycle, the foot approaches a selected foothold (a). Once in contact, the ground contact force in gravitational direction (force component $F_{z}$ in our setup) of the robot is increased (b1). After reaching the magnitude $F_{z \max }=25 \mathrm{~N}$, a tangential force $F_{T}=\left(\begin{array}{c}F_{x} \\ F_{y}\end{array}\right)$ is introduced and its magnitude is raised over time, while the tangential orientation of the force vector is altered (b2). The measurement process is aborted as soon as substantial foot slippage is detected or if the selected foothold could withstand its examination. To characterize the quality of the terrain samples, we introduce the simple metric $\mu_{F}=\frac{ \pm \sqrt{F_{x}^{2}+F_{y}^{2}}}{F_{z}} \quad \forall \quad F_{z}>0$. This value is similar to the coefficient of friction or even equal to the coefficient on flat ground, and a high value indicates a mechanically robust foothold. Together with the known $F_{z \max }$, this metric gives a conservative guess about the maximal allowed magnitudes of normal and tangential forces (with respect to a global horizontal plane) that can be applied at the examined foothold.

\section{E. Test setup}

To collect foothold quality estimates and geometric information of the terrain samples, the test-setup depicted in Figure 3 has been used. It is composed of a single robotic leg ([22]) with 3 degrees of freedom that is mounted to a fixed frame (a). To reduce the position error mainly determined by the backlash in the gears of the leg, a further camera was used to track the foot position. To reliably measure the ground contact forces, the leg was equipped with a force/torque sensor (ATI Mini45) that is integrated in the shank segment. The leg is controlled in two different modes: During swing phase, the leg is kinematically controlled. In ground contact, 


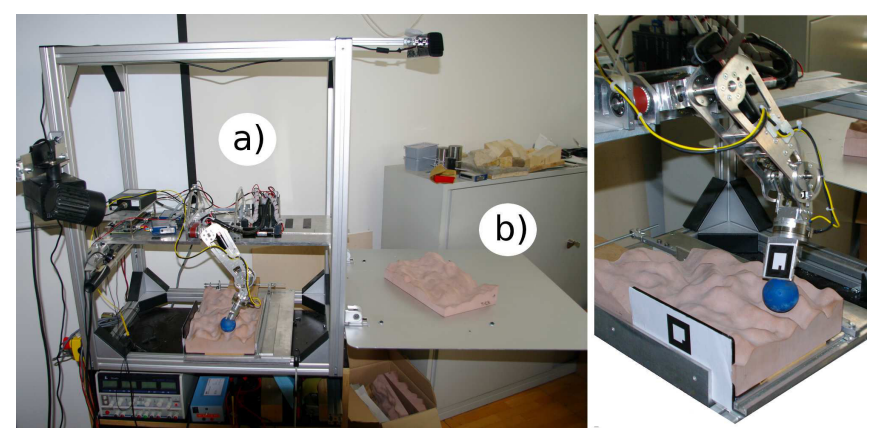

Fig. 3. Left side: A picture of the complete test-setup with the part a) to haptically explore the terrain samples and part b) to record the elevation map of the terrain samples. Right side: Close up of the robotic leg and the attached markers for the external position tracking. a)

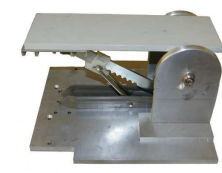

b)

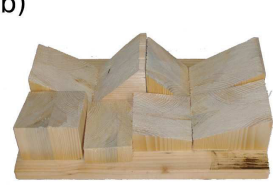

c)

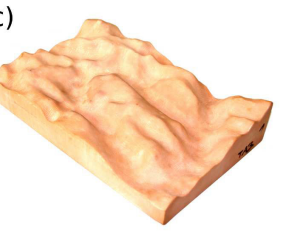

Fig. 4. Pictures of the three different terrain sample types for the experiments ( (a) adjustable incline for preliminary testing, (b) terrain sample with simple geometry and (c) naturally inspired sample with a complex shape.

force control is applied by adjusting the motor current as a function of the force sensor feedback. The foot element itself is a spherical rubber ball with a diameter of $4 \mathrm{~cm}$ and relatively high coefficient of friction. The testbench is further equipped with and Microsoft Kinect RGB-D-Camera to collect depth information of the terrain samples (b).

\section{EXPERIMENTS}

Experiments were conducted on three different types of terrain samples (Figure 4). First, to characterize the repeatability of the measurements under simplified conditions, an adjustable incline was used (a). The second sample type consisted of terrains with very simple geometry made of wooden blocks with different slope angles (b). The complex samples (c) were produced from NASA elevation data (Elevation data from the Shuttle Radar Topography Mission 30-arcsecond data (SRTM30)) and scaled so that the maximal difference in height was about double the foot diameter. In contrast to the other two sample types, the terrain were rapid prototyped with high precision. This allowed to compare the reconstructed terrain with the model data used for the production.

\section{RESUlTS}

\section{A. Geometric clustering}

The synthetic terrain data with a known cluster number (e.g. six in our case) was used to evaluate the three different clustering methods, K-means, K-medoid and Fuzzy c-means clustering. The data set was based on the features computed through the moments of the height map, feature set $\mathcal{F}_{2}$.

\begin{tabular}{|l|l|l|l|l|}
\hline $\mathrm{N}$ & $\mathrm{XB}$ & $\mathrm{CH}$ & $\mathrm{DI}$ & $\mathrm{S}$ \\
\hline \hline 3 & 7.4299 & 119.8584 & 0.70909 & $\mathbf{0 . 2 2 1 0 4}$ \\
\hline 4 & 7.4444 & 146.7819 & 0.4715 & 0.1536 \\
\hline 5 & 852.6972 & 14.1122 & 0.4715 & 0.19894 \\
\hline $\mathbf{6}$ & $\mathbf{8 5 7 . 1 2 0 1}$ & 9622.8888 & $\mathbf{9 . 3 5 0 2}$ & 0.16104 \\
\hline 7 & $\mathrm{NaN}$ & 2490.4064 & 0.043059 & 0.1421 \\
\hline 8 & $\mathrm{NaN}$ & 13105.8625 & 0.099595 & 0.12383 \\
\hline 9 & $\mathrm{NaN}$ & $\mathbf{9 4 2 6 6 . 0 2 4 8}$ & 0.04565 & 0.074619 \\
\hline & max & $\max$ & $\max$ & $\max$ \\
\hline
\end{tabular}

TABLE I

K-MEANS CLUSTERING

\begin{tabular}{|l|l|l|l|l|l|}
\hline $\mathrm{N}$ & $\mathrm{PC}$ & $\mathrm{CE}$ & $\mathrm{XB}$ & $\mathrm{DI}$ & $\mathrm{S}$ \\
\hline \hline 3 & 0.85152 & 0.28503 & 2.3459 & 0.70909 & 0.23575 \\
\hline 4 & 0.8126 & 0.35437 & 1.9359 & 0.71818 & $\mathbf{0 . 2 4 8 1 6}$ \\
\hline 5 & 0.89401 & 0.20238 & 3.4004 & 1.0188 & 0.12288 \\
\hline $\mathbf{6}$ & $\mathbf{0 . 9 9 9 2 8}$ & $\mathbf{0 . 0 0 3 1 5 3 3}$ & 3.5327 & $\mathbf{9 . 3 5 0 2}$ & 0.16489 \\
\hline 7 & 0.97237 & 0.04893 & 3.2895 & 0.12438 & 0.085801 \\
\hline 8 & 0.98047 & 0.033434 & $\mathbf{3 . 9 6 0 3}$ & 0.35471 & 0.12387 \\
\hline 9 & 0.9642 & 0.065387 & 3.6655 & 0.19966 & 0.089678 \\
\hline \multicolumn{6}{|c|}{ TABLE II } \\
\hline \multicolumn{7}{|c|}{ FuZZY C-MEANS CLUSTERING } & $\max$ & $\max$ & $\max$ \\
\hline
\end{tabular}

Table I contains the results for $K$-means clustering where seven different cluster sizes (from three to nine clusters) were proposed to the clustering method. While the CalinskiHarabasz $(\mathrm{CH})$ index as well as the Silhouette (S) index indicated an incorrect number of clusters for the given data set, the other validation methods, Xie-Bin (XB) and Dunn's index (DI), came up with the actual cluster number as optimal solution. The last row of the table indicates whether the maximum or minimum identifies the optimal number of clusters for the specified validation metric.

Table II shows the results of the validation metrics for the Fuzzy C-means clustering method. The Silhouette index once more indicated the wrong number of clusters, as well as the Xie-Bin method. All other methods identified the correct number of clusters. Assuming a similar structure of the underlying data, those results indicate which internal validation method perform best to determine the required cluster number to separate the data set.

To evaluate the effect of the different feature sets to represent the footholds, the clustering result (of the fuzzy clustering) are visualized by a Silhouette plot (Figure 5), where each data point is represented by a bar that ranges from -1 to +1 . Values close to +1 indicate that the data point is similar to others within the own cluster and distinct to points belonging to other clusters. It is obvious that data based on image statistic features is clearly separable, while the other two feature sets lead to data that is poorly separated.

\section{B. Validation of the repeatability of the quality measurement}

The experiments on the adjustable incline allowed to validate the proposed haptic exploration under very simple conditions. For each of the selected slope angles of the 

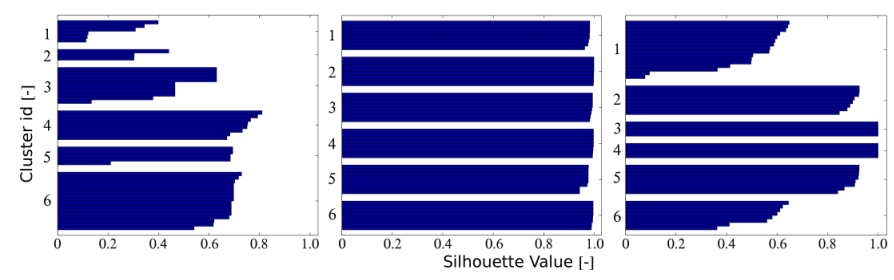

Fig. 5. Silhouette plots to visualize the results of the clustering process of the artificial foothold set (Left: Results for the feature set $\mathcal{F}_{1}$ that is based on the average slope angle, the relative height and the variance of the height. Middle: Results for the clustering on features based on the central moments (feature set $\mathcal{F}_{2}$ ). Left plot: Results for the Tamura features (feature set $\mathcal{F}_{3}$ ).

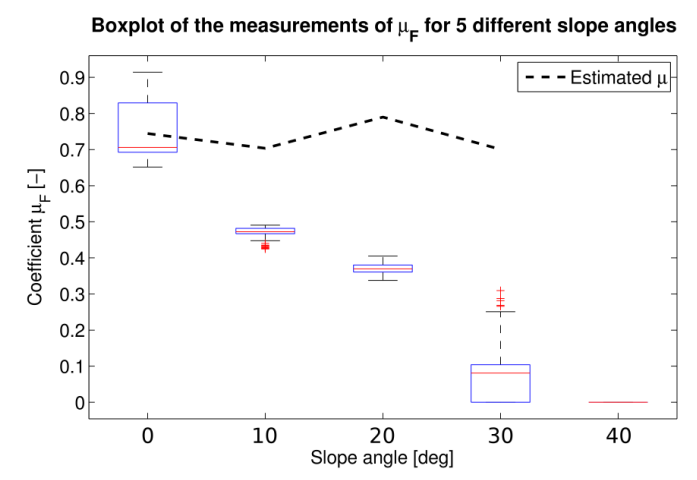

Fig. 6. Plot illustrating the distribution of the approximated $\mu_{F}$ for different angles of the adjustable incline. The dashed line represents the computed coefficient of friction $\mu$ of the foot/incline.

adjustable inclination (0,10,20,30, and 40 degrees $), 100$ measurement cycles were performed.

The box plot in Figure 6 illustrates the distribution of the measurements. Each box represents the data within the first and the third quartile, and the line the median of the approximated $\mu_{F}$. The whiskers of the plot point out the range of measurements that are still within \pm 1.5 times the interquartile range (difference of the third and the first quartile). Samples plotted as crosses mark the outliers. At 40 degrees, the leg started to slip before the tangential force could be applied for all the 100 measurements. As an indicator of the correctness of the measurements, the coefficient of friction $\mu$ of the adjustable incline with angle $\alpha$ was calculated: $\mathbf{F}^{\prime}=\mathbf{R} \tilde{\mathbf{F}}$, so that $\mu=\frac{\sin (\alpha)+\cos (\alpha) \cdot \mu_{F}}{\cos (\alpha)-\sin (\alpha) \cdot \mu_{F}}$, where the vector $\mathbf{F}^{\prime}$ denotes the force components normal/perpendicular to the slope, $\mathbf{R}$ the $2 \mathrm{D}$ rotation matrix and $\tilde{\mathbf{F}}$ the measured force components in robot body coordinates. Ideally, when slipping in the direction of the slope, the computed $\mu$ should be identical for all the inclination angles. The dashed line in Figure 6 shows a plot of the computed friction coefficient for the slope angles smaller than 40 degrees.

\section{Real terrain samples}

Figure 7 illustrates the haptically measured foothold quality at the different locations of the simple (left) and the complex (right) ground geometry sample. We will refer to this as haptic ground truth data. As expected, flat ground and concave structures show the best quality. Furthermore, also hilltop areas represent good quality as the relatively
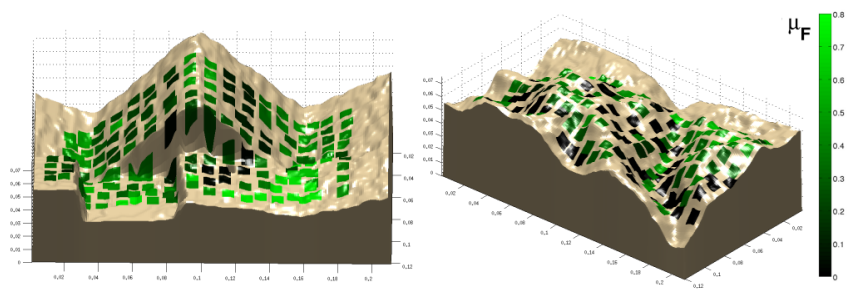

Fig. 7. Plot of the terrain samples and the quality metric for the footholds based on the haptic exploration (used as ground truth) (Left: Terrain with simple geometry, right: naturally inspired terrain).

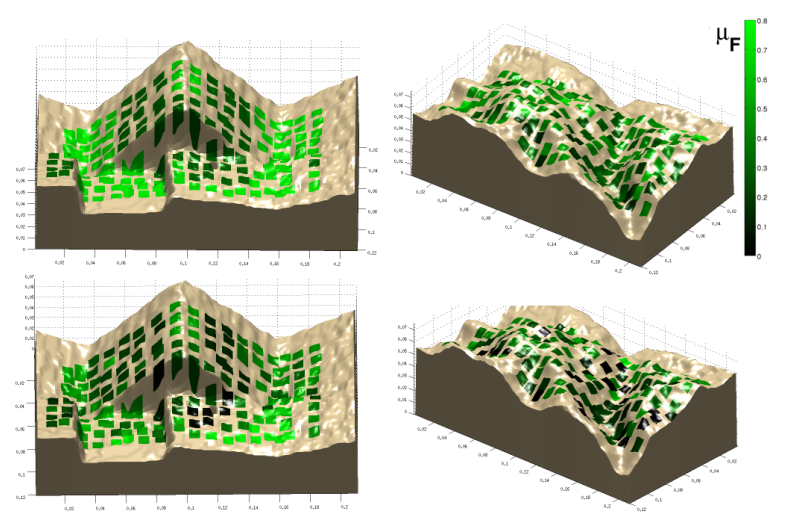

Fig. 8. Plot of the terrain for testing with the predicted quality measure (Top left plot: 4 clusters, bottom left: 19 clusters, top right: 25 cluster, bottom right: 46 clusters).

compliant ball foot deforms such that high tangential forces can be applied.

We further use the k-means algorithm to cluster the footholds according the terrain geometry and assign a single haptic quality measure to each cluster. This quality 'label' is determined by computing the mean quality measure of all the explored footholds of the corresponding cluster (one half of the ground truth footholds were used for this quality labeling process). The determination of the cluster number (19 for the simpler terrain and 46 for the naturally inspired terrain) is based on the interpretation of the Dunn's index as internal cluster validation method.

The top left plot of Figure 8 shows the testing results for a cluster number of four. The mean absolute error of the foothold quality estimate and ground truth is about 0.2592 , and is reduced to 0.1042 for 19 clusters. The right side of the Figure 8 shows the same for the terrain with naturally inspired geometry. Here, if 25 clusters are used, the mean error of $\mu_{F}$ is about 0.0996. Figure 9 visualizes the results of a robustness prediction for a haptically unexplored shape. The blue regions are unknown - no haptic label could be associated to the shape of the region (the ground truth footholds were used for this quality labeling process). Intuitively, the results of the prediction seem to make sence - flat regions are more robust, the robustness decreases with increasing slope angle at the boarders and the unknown footholds are not represented in the ground truth data. 


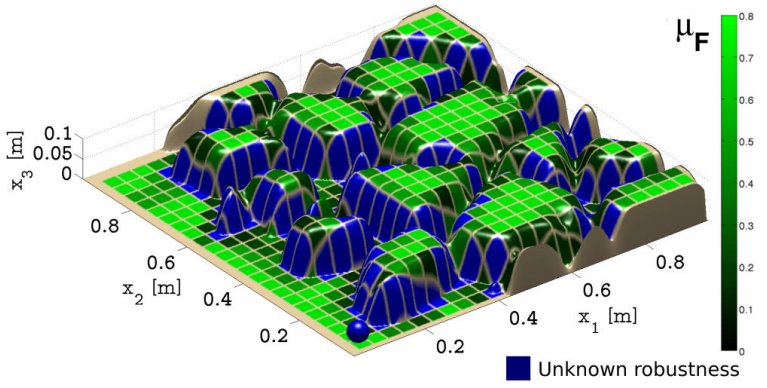

Fig. 9. Visualisation of the robustness prediction for a 'haptically unknown' terrain $\left(1 \mathrm{~m}^{2}\right)$.

\section{CONCLUSION}

In this paper, an unsupervised method to estimate the foothold quality by only knowing the foothold geometry has been presented. First, possible footholds are clustered according to features that are based on geometrical properties of the terrain. Second, haptic information that is recorded during ground contact of a robotic leg is assigned to the clusters (sets of footholds). In the study, three different sets of features, three different clustering algorithms and various cluster validation methods were examined based on synthetic and real terrain data. The synthetic data has been used to evaluate the usefulness of the feature sets as well as the cluster validation methods. This helped to identify feature sets that are invariant to rotation and to a certain level of noise. Features based on the central moments (feature set $\mathcal{F}_{2}$ ) of the elevation map proved to describe the applied data set most accurate. Further, a method has been presented to evaluate the quality of footholds without human intervention or expert knowledge. Instead, the quality of a potential foothold is determined by the experience from foot/ground interaction of the robot. The method has been validated repeatedly on a adjustable incline with low variance. Geometric foothold clustering and haptic quality identification was finally successfully applied to different simple and complex geometries. Even if experiments with real terrains and real robotic legs have been performed, so far, the effect of different mechanical terrain material parameters (such as shear strength, coefficient of friction or ductility) are neglected. Further investigation will be required to address this subject.

In comparison to existing work, the presented method is not based on human intuition but generates foothold labels that directly correlate to the maximal forces that can be applied without slipping. This renders the method especially useful for unknown terrain, where the mechanical robustness can not be determined by human experts anymore. This illustrates that a robot-centric perspective on foothold quality can be highly advantageous and emphasizes the usefulness of the approach.

\section{ACKNOWLEDGments}

The authors gratefully acknowledge the team of the Raplab of D-ARCH, Alessandro Tellini, Reto Klingenfuss, and
Daniel Bachmann for the great work in manufacturing of the terrain samples and Markus Buehler as well as Dario Fenner for the support on the mechanical construction.

\section{REFERENCES}

[1] M. P. Murphy, A. Saunders, C. Moreira, A. A. Rizzi, and M. Raibert, "The LittleDog robot", in International Journal of Robotics Research, 30(2), pp. 145-149, 2010.

[2] J. R. Rebula, P. D. Neuhaus, B. V. Bonnlander, M. J. Johnson, J. E. Pratt, "A Controller for the LittleDog Quadruped Walking on Rough Terrain", in IEEE International Conference on Intelligent Robots and Systems, 2007.

[3] M.Kalakrishnan, J. Buchli, P. Pastor, S. Schaal, "Learning Locomotion over Rough Terrain using Terrain Templates", in IEEE International Conference on Intelligent Robots and Systems, 2009.

[4] M. Zucker, J. A. Bagnell, C. G. Atkeson, and J. Kuffner, "An Optimization Approach to Rough Terrain Locomotion", in IEEE International Conference on Robotics and Automation, 2010.

[5] A. Krebs, C. Pradalier, R. Siegwart, "Adaptive Rover Behavior Based on Online Empirical Evaluation: Rover-Terrain Interaction and Nearto-Far Learning", in Journal of Field Robotics, Vol. 27, No. 2, pp 158-180, 2010.

[6] C. Weiss, H. Fröhlich and Andreas Zell, "Vibration-based terrain classification using Support Vector Machines", in International Conference on Intelligent Robots and Systems, 2006.

[7] C.A. Brooks, K.D. Iagnemma, "Self-Supervised Classification for Planetary Rover Terrain Sensing", in IEEE Aerospace Conference 2007

[8] L. Ojeda, J. Borenstein, G. Witus and R. Karlsen, "Terrain Characterization and Classification with a Mobile Robot", in Journal of Field Robotics, Vol. 23, No. 2, pp. 103-122, 2005.

[9] M. Hoepflinger, C.D. Remy, M. Hutter, S. Haag and R. Siegwart, "Haptic Terrain Classification on Natural Terrains for Legged Robots", in Proc. of the 13th International Conference on Climbing and Walking Robots (CLAWAR), 2010

[10] M. Hoepflinger, C.D. Remy, M. Hutter, L. Spinello, and R. Siegwart, "Haptic Terrain Classification for Legged Robots", in IEEE International Conference on Robotics and Automation, 2010.

[11] Bajracharya, M. and Benyang Tang and Howard, A. and Turmon, M. and Matthies, L., "Learning long-range terrain classification for autonomous navigation", in IEEE International Conference on Robotics and Automation, 2008.

[12] M. Halkidi, Y. Batistakis, and M. Vazirgiannis, "On Clustering Validation Techniques", in Journal of Intelligent Information Systems, Vol.17 (2-3), pp. 107-145, 2001

[13] J. Z. Kolter, M. P. Rodgers, and A. Y. Ng, "A control architecture for quadruped locomotion over rough terrain", in IEEE International Conference on Robotics and Automation, 2009.

[14] H. Tamura, S. Mori, T. Yamawaki, "Textural Features Corresponding to Visual Perception", in IEEE Transaction on Systems, Man, and Cybernetcs, Vol. SMC-8, No. 6, pp. 460-472, 1978.

[15] J. Dunn, "Well separated clusters and optimal fuzzy partitions", Journal of Cybernetics, Vol. 4, No. 1, pp. 95-104, 1974.

[16] L. Yanchi, L. Zhongmou, X. Hui, G. Xuedong, W. Junjie, "Understanding of Internal Clustering Validation Measures", IEEE International Conference on Data Mining, pp. 911-916, 2010.

[17] A. K. Jain, M. N. Murty, and P. J. Flynn, "Data Clustering: A Review", 1999

[18] R. B. Calinski and J. Harabasz, "A dendrite method for cluster analysis", Communications in Statistics - Theory and Methods, Vol. 3, pp. 1-27, 1974.

[19] P. Rousseeuw, "Silhouettes: a Graphical Aid to the Interpretation and Validation of Cluster Analysis", Journal of Computational and Applied Mathematics, pp. 53-65, 1987.

[20] X. L. Xie and G. Beni, "A validity measure for fuzzy clustering", IEEE Transactions on Pattern Analysis and Machine Intelligence, Vol. 13, No. 8, pp. 841-847, 1991.

[21] J.C. Bezdek and J.C. Dunn , "Optimal fuzzy partitions: A heuristic for estimating the parameters in a mixture of normal dustrubutions", IEEE Transactions on Computers, pp. 835-838, 1975.

[22] Remy, C. David; Baur, Oliver; Latta, Martin; Lauber, Andi; Hutter, Marco; Hoepflinger, Mark A.; Pradalier, Cdric; Siegwart, Roland, "Walking and crawling with ALoF: a robot for autonomous locomotion on four legs", in Industrial Robot, Vol. 38, pp. 264-268, 2011. 